\title{
Antiinflammatory Activity of Triazine Thiazolidinone Derivatives: Molecular Docking and Pharmacophore Modelling
}

\author{
R. S. SHINDE*, V. H. MASAND ${ }^{1}$ AND M. K. PATIL ${ }^{2}$ \\ Department of Chemistry, Dayanand Science College, Latur-413 512, ${ }^{1}$ Department of Chemistry, Vidya Bharati College, \\ Amaravati-444 602, ${ }^{2}$ Department of Chemistry, Dr. Babasaheb Ambedkar Marathwada University, Aurangabad, Sub-Campus \\ Osmanabad-413 501, India
}

\section{Shinde et al.: Antiinflammatory Activity of Triazine Thiazolidinone Derivatives}

\begin{abstract}
Some 3-(4,6-dichloro-1,3,5-triazin-2-yl)-2-phenylthiazolidin-4-one derivatives were prepared by cyclocondensation reaction between 2 -amino-4,6-dichloro-1,3,5-triazine, substituted aromatic aldehyde and ethyl-2-mercaptoacetate, with an yield in the range 76-86 \%. Prepared compounds showed antiinflammatory activity. The halogenated electron-withdrawing groups on the phenyl ring of 4-thiazolinone generated antiinflammatory activity. Among the synthesized compounds, 3-(4,6-dichloro-1,3,5-triazin-2-yl)-2-(2,5difluorophenyl)thiazolidin-4-one showed better antiinflammatory activity with 72 and $79 \%$ inhibition for TNF- $\alpha$ and IL-6, respectively. Also, molecular docking and pharmacophore modelling performed for this active antiinflammatory compound highlighted that hydrophobicity as an important feature for activity optimization.
\end{abstract}

Key words: Triazine thiazolidinone, antiinflammatory activity, molecular docking, pharmacophore modeling

Medicinal chemistry has an important role in medicine and health care. The leading objective of medicinal chemistry is to design and synthesize therapeutically potential molecules, study their pharmacokinetic and pharmacodynamic properties, and thoroughly test them as human therapeutic agents. It has been established that infections due to multidrug-resistance (MDR) microbes is a serious concern worldwide and an important issue, also. According to AR threat report in 2013, in the USA annually, at least two million infections and 23000 deaths occurred ${ }^{[1]}$ and in the latest report, according to WHO's new Global Antimicrobial Surveillance System, there is an extensive existence of antibiotic resistance among 500000 people with suspected bacterial infections across 22 countries $^{[2]}$.

The solution for this is actively look for new drugs with newer mechanisms of action. In this context, fundamentally the focus should be on understanding the principles and ecological factors, which can affect MDR in bacteria ${ }^{[3,4]}$. Rapid growth of MDR strains of pathogens result in a severe resistance development towards presently available antimicrobial drugs in the market.
In spite of several efforts during the last few decades to develop new antimicrobials, the efforts were not completely successful to control the rapid upsurge of resistance genes evolving among both Gram-positive and Gram-negative pathogens ${ }^{[5]}$. In this regard, there is an urgent need to develop new and improved antimicrobial drugs. Antimicrobial research is important not only for development of effective treatments but also for the increased business potential of the pharma industry ${ }^{[4,6]}$. Recently thiazole, a five-membered ring heterocycle with three carbons, one sulphur atom and one nitrogen atom; and its derivatives thiazolidinones having carbonyl group at 2, 4 or 5-position have been a focus of therapeutic interest ${ }^{[7,8]}$. It has been revealed by Palekar et al..$^{[9]}$, Hamama et al. ${ }^{[10]}$, Kumar et al. ${ }^{[11]}$, Kaminskyy et al. ${ }^{[12]}$, Samadhiya et al. ${ }^{[13]}$ and others ${ }^{[14]}$ that in different synthetic drugs pharmacological activities are associated with the thiazolidinone ring

This is an open access article distributed under the terms of the Creative Commons Attribution-NonCommercial-ShareAlike 3.0 License, which allows others to remix, tweak, and build upon the work non-commercially, as long as the author is credited and the new creations are licensed under the identical terms

Accepted 30 July 2019

Revised 26 April 2019

Received 29 December 2018

Indian J Pharm Sci 2019;81(5):851-858 
system as a core structure. Substituents at 2, 3 and 5-position are useful for varying the properties but greatest difference in structure and properties has been exerted by groups attached to carbon atom in 2-position ${ }^{[15]}$. As the thiazolidinone possesses almost all different types of pharmacological activities, it has been considered as supernatural moiety or magic moiety i.e. a wonder nucleus ${ }^{[16-19]}$. On the other hand, triazine derivatives are another important class of heterocycles having wide range of biological activities including antimicrobial, anticancer, antimalarial, antiviral, antitubercular and antiHIV ${ }^{[20]}$.

Furthermore, several systems of heterocyclic compounds having triazine linked-thiazolidinones have been synthesized and their pharmacological activities were examined ${ }^{[21-23]}$. The thiazolidinones bearing triazine analogues have been found to be potential bioactive molecules ${ }^{[24-27]}$. Therefore, it was thought worthwhile to develop a scheme which combines biolabile nuclei, 1,3,5-triazine and thiazolidinones together in a molecular framework so that their additive effect can increase already existing antimicrobial potential. In line with this, our earlier work on aryl substituted 3-(4,6-dichloro-1,3,5-triazin2-yl)thiazolidin-4-ones contributed for evaluation of the antibacterial and antimicrobial activity ${ }^{[28]}$.

In the present work, aryl substituted 3-(4,6-dichloro1,3,5-triazin-2-yl)thiazolidin-4-ones (fig. 1) were synthesized from aromatic aldehyde, 4,6-dichloro1,3,5-triazin-2-amine and ethyl 2-mercaptoacetate using dry ethanol. The structure of these compounds was confirmed on the basis of ${ }^{1} \mathrm{H}$ nuclear magnetic resonance (NMR), and mass spectra. Antiinflammatory activity of all the synthesized compounds was screened. In the structure activity relationship (SAR) study, the biological activity of these molecules was compared with that of a reference compound. Furthermore, in recent years, different computational techniques have gained popularity to get insight into the mechanism of action of small molecules and streamline the future optimizations to achieve required objectives. Therefore, molecular docking, which is a structure-based drug design technique study was performed to determine the pharmacophore model (ligand-based drug design) to understand the key structural features, which could help in further optimization of triazine-thiazolidinone derivatives to obtain better drug candidates.

\section{MATERIAL AND METHODS}

For the synthesis of 3-(4,6-dichloro-1,3,5-triazin-2- yl)-2-phenylthiazolidin-4-one derivatives, 2-amino4,6-dichloro-1,3,5-triazine, substituted aromatic aldehyde and ethyl-2-mercaptoacetate were obtained from commercial sources. The solvent dry ethanol was purified by distillation before use. IR spectra were recorded on a Shimadzu FT-IR-8400 instrument using $\mathrm{KBr}$ pellet method at the Department of Chemistry, Dayanand College, Solapur. Mass spectra were recorded on a Shimadzu GC-MS-QP-2010 model using direct injection probe technique and the ${ }^{1} \mathrm{H}$ NMR was determined in $\mathrm{CDCl}_{3}$ solution on a Bruker AscendTM 400MHz-NMR spectrometer at S.A.I.F. Division, Indian Institute of Technology, Bombay.

\section{Synthesis of 3-(4,6-dichloro-1,3,5-triazin-2-yl)-2-} phenyl thiazolidin-4-one (4a):

The synthesis of the target compounds was accomplished via one-pot multicomponent reaction. A mixture of 2-amino 4,6-dichloro-1,3,5-triazine (1, 1 mmol, $0.164 \mathrm{~g}$ ), benzaldehyde (3, $1 \mathrm{mmol}, 0.106 \mathrm{~g}$ ) and ethyl-2-mercaptoacetate $(2,1 \mathrm{mmol}, 0.120 \mathrm{~g})$ was refluxed for $2 \mathrm{~h}$ in dry ethanol $(20 \mathrm{ml})$ as reported in our previous article ${ }^{[28]}$. The progress of reaction was monitored by TLC (toluene:acetone, 4:6). The excess alcohol was evaporated in the vacuum. The resulting crude product was added to crush ice. The solid obtained was washed with water, dried and recrystallized from ethanol to afford final respective compounds (4a). Similarly remaining compounds (4b-1) were prepared. The synthetic strategy for novel compounds triazinebased thiazolidinones is outlined in fig. 2. The structure of all synthesized compounds was confirmed on the basis of ${ }^{1} \mathrm{H}$ NMR, mass spectra and elemental analysis. The products were further ascertained by physical and analytical analyses.

\section{Spectral data:}

3-(4,6-dichloro-1,3,5-triazin-2-yl)-2-phenylthiazolidin -4-one (4a), pale yellow solid; yield- $80 \%$, melting

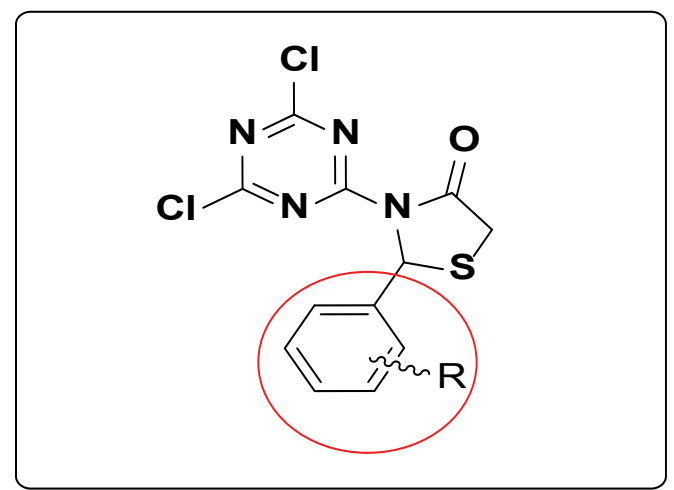

Fig. 1: Design scaffold

Indian Journal of Pharmaceutical Sciences 


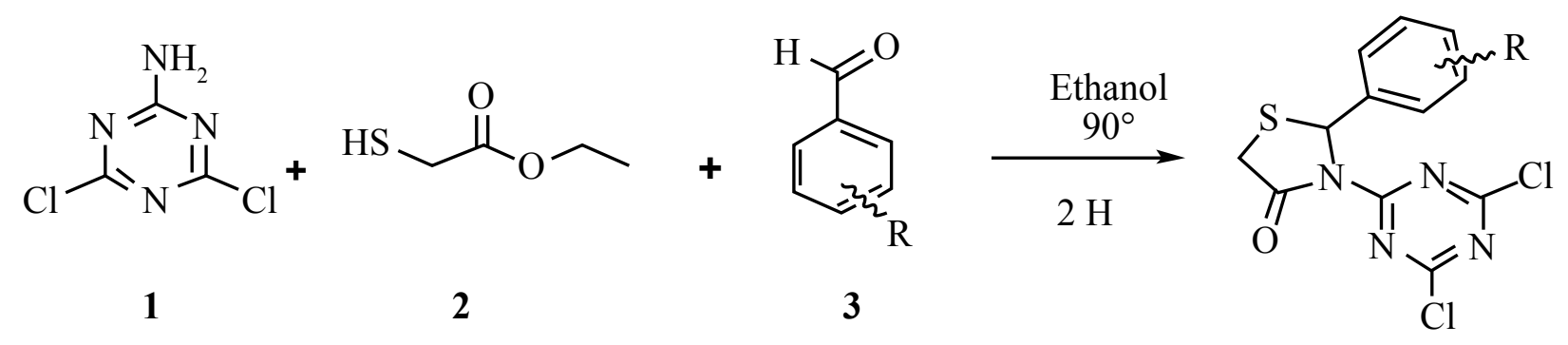

4a-1

Fig. 2: Synthesis of aryl substituted 3-(4,6-dichloro-1,3,5-triazin-2-yl)thiazolidin-4-one derivatives $\mathrm{R}=4 \mathrm{a}-\mathrm{l} ; \mathbf{4 a}=$ phenyl, $4 \mathrm{~b}=4$-methoxy, $4 \mathrm{c}=2$-fluoro, $4 \mathrm{~d}=\mathbf{2}$-fluoro-4-bromo, 4e=2-bromo, $4 \mathrm{f}=2$-fluoro-5-chloro, $4 \mathrm{~g}=3$-methyl, $4 \mathrm{~h}=4$-nitro, $4 i=2$-hydroxy, $4 j=3$-methoxy, $4 \mathrm{k}=2,5$-difluoro, $4 \mathrm{l}=3-\mathrm{CN}$

point (mp)- 138-140.${ }^{1} \mathrm{H}$ NMR $\left(400 \mathrm{MHz}, \mathrm{CDCl}_{3}\right): \delta$ (ppm) 7.41-6.85 (m, 5H), $5.33(\mathrm{~s}, 1 \mathrm{H}), 3.40(\mathrm{~d}, J=7.1$ $\mathrm{Hz}, 2 \mathrm{H}) . \mathrm{IR}(\mathrm{KBr}): v\left(\mathrm{~cm}^{-1}\right) 2842(\mathrm{C}-\mathrm{H}$ str. in aromatic ring), $1723(\mathrm{C}=\mathrm{O}$ of thiazolidinone $), 1660(-\mathrm{C}=\mathrm{C}-$ str. in aromatic ring), 814 (C-N-str. in s-triazine). MS (70 eV) $m / z: 325.96\left[\mathrm{M}^{+} 100 \%\right], 327.96[\mathrm{M}+2,66.66 \%]$, $329.96[\mathrm{M}+4,11.11 \%]$. Anal. calcd. for $\mathrm{C}_{12} \mathrm{H}_{8} \mathrm{Cl}_{2} \mathrm{~N}_{4} \mathrm{OS}$ : C, 44.05; H, 2.46; N, 17.12: S, 9.80; found: C, 43.98; H, 2.35; N, 17.00: S, $9.60 \%$.

3-(4,6-dichloro-1,3,5-triazin-2-yl)-2-(4-methoxy phenyl)thiazolidin-4-one (4b), pale yellow solid; yield$82 \%$, mp- 140-142 ${ }^{\circ}{ }^{1} \mathrm{H}$ NMR (400 MHz, $\mathrm{CDCl}_{3}$ ): $\delta$ (ppm) $7.41(\mathrm{~m}, 2 \mathrm{H}), 6.89(\mathrm{~m}, 2 \mathrm{H}), 5.34(\mathrm{~d}, 1 \mathrm{H}), 3.82(\mathrm{~s}$, $3 \mathrm{H}), 3.43(\mathrm{~d}, J=7.2 \mathrm{~Hz}, 2 \mathrm{H}) . \mathrm{IR}(\mathrm{KBr}): v\left(\mathrm{~cm}^{-1}\right) 2838(\mathrm{C}-\mathrm{H}$ str. in aromatic ring), $1720(\mathrm{C}=\mathrm{O}$ of thiazolidinone $)$, $1655(-\mathrm{C}=\mathrm{C}-$ str. in aromatic ring), $813(\mathrm{C}-\mathrm{N}-\mathrm{str}$. in s-triazine). MS (70 eV) m/z: $355.98\left[\mathrm{M}^{+}, 100 \%\right]$, $357.98[\mathrm{M}+2,66.66 \%], 358.98[\mathrm{M}+3,11.66 \%]$, anal. calcd. for $\mathrm{C}_{13} \mathrm{H}_{10} \mathrm{Cl}_{2} \mathrm{~N}_{4} \mathrm{O}_{2} \mathrm{~S}: \mathrm{C}, 43.71 ; \mathrm{H}, 2.82 ; \mathrm{N}$, 15.68: S, 8.98; found: C, 43.30; H, 2.45; N, 15.48: S, $8.40 \%$.

3-(4,6-dichloro-1,3,5-triazin-2-yl)-2-(2-fluorophenyl) thiazolidin-4-one (4c): pale yellow solid, yield- $78 \%$, mp- $145-147^{\circ} .{ }^{1} \mathrm{H}$ NMR (400 MHz, $\mathrm{CDCl}_{3}$ ): $\delta(\mathrm{ppm})$ $7.58(\mathrm{td}, J=7.5,1.9 \mathrm{~Hz}, 1 \mathrm{H}), 7.32(\mathrm{td}, J=6.6,5.8$, $3.7 \mathrm{~Hz}, 1 \mathrm{H}), 7.18$ (td, $J=7.6,1.2 \mathrm{~Hz}, 1 \mathrm{H}), 7.06$ (ddd, $J=9.5,8.3 .1 .2 \mathrm{~Hz}, 1 \mathrm{H}, \mathrm{Ar}-\mathrm{H}), 5.94(\mathrm{~d}, J=7.4 \mathrm{~Hz}, 1 \mathrm{H})$, $4.25-4.10(\mathrm{~m}, 2 \mathrm{H})$. IR (KBr): $v\left(\mathrm{~cm}^{-1}\right) 2836(\mathrm{C}-\mathrm{H}$ str. in aromatic ring), $1715(\mathrm{C}=\mathrm{O}$ of thiazolidinone $)$, 1629 (-C=C- str. in aromatic ring), 809 (C-N- str. in s-triazine). MS (70 eV) m/z: $343.90\left[\mathrm{M}^{+}, 100 \%\right]$, 345.90 [M+2, $66.66 \%$ ], 347.11 [M+4, $11.11 \%]$, anal. calcd for $\mathrm{C}_{12} \mathrm{H}_{7} \mathrm{~F} \mathrm{Cl}_{2} \mathrm{~N}_{4} \mathrm{OS}$ : C, 41.75; H, 2.04; N, 16.23: S, 9.29; found: C, 41.55; H, 2.00; N, 16.10: S, $9.10 \%$.

2-(4-bromo-2-fluorophenyl)-3-(4,6-dichloro-1,3,5triazin-2-yl)thiazolidin-4-one (4d): pale yellow solid, yield- $76 \%$, mp- $145-147^{\circ} .{ }^{1} \mathrm{H}$ NMR (400 MHz, $\left.\mathrm{CDCl}_{3}\right): \delta(\mathrm{ppm}) 7.48-7.42(\mathrm{~m}, 1 \mathrm{H}), 7.33(\mathrm{dd}, J=8.4$, $2.0 \mathrm{~Hz}, 1 \mathrm{H}), 7.25$ (dd, $J=9.4,1.9 \mathrm{~Hz}, 1 \mathrm{H}), 5.86$ (s, 1H), 4.20 - 4.13 (m, 2H). IR (KBr): $v\left(\mathrm{~cm}^{-1}\right) 2830(\mathrm{C}-\mathrm{H}$ str. in aromatic ring), $1719(\mathrm{C}=\mathrm{O}$ of thiazolidinone $)$, $1650(-\mathrm{C}=\mathrm{C}-\mathrm{str}$. in aromatic ring), $810(\mathrm{C}-\mathrm{N}-\mathrm{str}$. in s-triazine) $\mathrm{cm}^{-1}$. MS (70 eV) $\mathrm{m} / z: 424.8\left[\mathrm{M}^{+}, 60 \%\right]$, $424.8[\mathrm{M}+2,100 \%], 424.8[\mathrm{M}+4,46 \%], 424.8[\mathrm{M}+6$, $6 \%$ ], anal. calcd for $\mathrm{C}_{12} \mathrm{H}_{6} \mathrm{~F} \mathrm{Br} \mathrm{Cl}_{2} \mathrm{~N}_{4} \mathrm{OS}$ : C, 33.99; $\mathrm{H}$, 1.43; N, 13.21: S, 7.56; found: C, 33.41; H, 1.35; N, 13.10: S, $7.20 \%$.

2-(4-bromophenyl)-3-(4,6-dichloro-1,3,5-triazin-2-yl) thiazolidin-4-one (4e), pale yellow solid, yield- $80 \%$, mp- $160-162^{\circ} .{ }^{1} \mathrm{H}$ NMR (400 MHz, $\left.\mathrm{CDCl}_{3}\right): \delta(\mathrm{ppm})$ 7.53-7.47 (m, 2H), 7.41-7.34 (m, 2H), $5.34(\mathrm{~s}, 1 \mathrm{H})$, 4.17 (q, $J=7.2 \mathrm{~Hz}, 2 \mathrm{H})$. IR (KBr): $v\left(\mathrm{~cm}^{-1}\right) 2830(\mathrm{C}-\mathrm{H}$ str. in aromatic ring), $1721(\mathrm{C}=\mathrm{O}$ of thiazolidinone), $1656(-\mathrm{C}=\mathrm{C}-$ str. in aromatic ring), $811(\mathrm{C}-\mathrm{N}-$ str. in s-triazine). MS (70 eV) m/z: $403.86\left[\mathrm{M}^{+}, 60 \%\right], 405.86$ $[\mathrm{M}+2,100 \%], 407.86[\mathrm{M}+4,46 \%], 409.86[\mathrm{M}+6$, $6 \%$ ], anal. calcd. for $\mathrm{C}_{12} \mathrm{H}_{7} \mathrm{BrCl}_{2} \mathrm{~N}_{4} \mathrm{OS}$ : $\mathrm{C}, 35.49 ; \mathrm{H}$, $1.74 ; \mathrm{N}, 13.80 ; \mathrm{S}, 7.90$, found: $\mathrm{C}, 35.30 ; \mathrm{H}, 1.45 ; \mathrm{N}$, $13.48 ; \mathrm{S}, 7.20 \%$.

3-(4,6-dichloro-1,3,5-triazin-2-yl)-2-(4-chloro-2fluorophenyl) thiazolidin-4-one (4f), pale yellow solid, yield- 81\%; mp: $155-157^{\circ}$. ${ }^{1} \mathrm{H}$ NMR (400 $\mathrm{MHz}, \mathrm{CDCl}_{3}$ ): $\delta$ (ppm) $7.69(\mathrm{dd}, J=6.4,2.6 \mathrm{~Hz}, 1 \mathrm{H})$, 7.41 (ddd, $J=8.7,4.6,2.6 \mathrm{~Hz}, 1 \mathrm{H}), 6.95$ (dd, $J=9.6$, $8.7 \mathrm{~Hz}, 1 \mathrm{H}), 5.86(\mathrm{~s}, 1 \mathrm{H}), 4.17(\mathrm{~m}, 2 \mathrm{H})$. IR (KBr): v $\left(\mathrm{cm}^{-1}\right) 2820(\mathrm{C}-\mathrm{H}$ str. in aromatic ring), $1720(\mathrm{C}=\mathrm{O}$ of thiazolidinone), 1651 (-C=C-str. in aromatic ring), 812 (C-N- str. in s-triazine). MS $(70 \mathrm{eV}) \mathrm{m} / z: 377.93\left[\mathrm{M}^{+}\right.$, $100 \%$ ], 379.93 [M+2, $100 \%$ ], 381.93 [M+4, $33 \%$ ], $383.93\left[\mathrm{M}+6,4 \%\right.$ ], anal. calcd. for $\mathrm{C}_{12} \mathrm{H}_{6} \mathrm{FCl}_{3} \mathrm{~N}_{4} \mathrm{OS}: \mathrm{C}$, 37.97; H, 1.59 ; N, 14.76: S, 8.45; found: C, 37.41; H, 1.35; N, 14.30: S, 8.20\%. 
3-(4,6-dichloro-1,3,5-triazin-2-yl)-2-m-tolyl thiazolidin-4-one $(4 \mathrm{~g})$, pale yellow solid, yield- $86 \%$, mp- $140-142^{\circ} .{ }^{1} \mathrm{H}$ NMR $\left(400 \mathrm{MHz}, \mathrm{CDCl}_{3}\right): \delta(\mathrm{ppm})$ 7.37-7.33(m, 2H), 7.32-7.27 (m, 2H), $5.88(\mathrm{~s}, 1 \mathrm{H})$, $2.21(\mathrm{~s}, 3 \mathrm{H}), 4.22-4.27$ (d, J=7.2 Hz, 2H). IR (KBr): v $\left(\mathrm{cm}^{-1}\right) 2842(\mathrm{C}-\mathrm{H}$ str. in aromatic ring), $1718(\mathrm{C}=\mathrm{O}$ of thiazolidinone), 1660 (-C $=\mathrm{C}$ - str. in aromatic ring), 814 (C-N- str. in s-triazine). MS $(70 \mathrm{eV}) \mathrm{m} / \mathrm{z}: 340.6\left[\mathrm{M}^{+}\right.$, $100 \%$ ], 342.6 [M+2, $66.66 \%$ ], 344.6 [M+4, $11.11 \%$ ], anal. calcd. for $\mathrm{C}_{13} \mathrm{H}_{10} \mathrm{Cl}_{2} \mathrm{~N}_{4} \mathrm{OS}$ : C, 45.76; $\mathrm{H}, 2.95 ; \mathrm{N}$, 16.42: S, 9.40; found: C, 45.56; H, 2.65; N, 16.10: S, $9.30 \%$.

3-(4,6-dichloro-1,3,5-triazin-2-yl)-2-(4-nitrophenyl) thiazolidin-4-one (4h), pale yellow solid, yield- $78 \%$, mp- $170-172^{\circ} .{ }^{1} \mathrm{H}$ NMR $\left(400 \mathrm{MHz}, \mathrm{CDCl}_{3}\right): \delta(\mathrm{ppm})$ $7.66(\mathrm{~m}, 2 \mathrm{H}), 6.85(\mathrm{~m}, 2 \mathrm{H}), 5.84(\mathrm{~s}, 1 \mathrm{H}), 4.19-4.12$ $(\mathrm{m}, 2 \mathrm{H})$. IR $(\mathrm{KBr}): v\left(\mathrm{~cm}^{-1}\right) 2836(\mathrm{C}-\mathrm{H}$ str. in aromatic ring), $1721(\mathrm{C}=\mathrm{O}$ of thiazolidinone $), 1641(-\mathrm{C}=\mathrm{C}-\mathrm{str}$. in aromatic ring), 807 (C-N- str. in s-triazine). MS (70 eV) $m / z$ : $370.8\left[\mathrm{M}^{+}, 100 \%\right], 372.8$ [M+2, $\left.66.66 \%\right]$, $374.8[\mathrm{M}+4,11.11 \%]$, anal. calcd. for $\mathrm{C}_{12} \mathrm{H}_{7} \mathrm{Cl}_{2} \mathrm{~N}_{5} \mathrm{O}_{3}$ S: C, 38.72 ; H, 1.90 ; N, 18.82: S, 8.62; found: C, $38.41 ; \mathrm{H}, 1.65 ; \mathrm{N}, 18.40: \mathrm{S}, 8.40 \%$.

3-(4,6-dichloro-1,3,5-triazin-2-yl)-2-(2-hydroxy phenyl)thiazolidin-4-one (4i), pale yellow solid, yield$82 \%$, mp- 160-162 ${ }^{\circ}$ ' $\mathrm{H}$ NMR (400 MHz, $\left.\mathrm{CDCl}_{3}\right)$ : $\delta$ (ppm) 7.58 (d, $J=11 \mathrm{~Hz}, 1.8 \mathrm{~Hz}, 1 \mathrm{H}), 7.32$ (d, $J=6.5$, 5.7, 3.6 Hz, 1H), 7.18 (d, J=7.5, $1.3 \mathrm{~Hz}, 1 \mathrm{H}), 7.06$ (dd, $J=9.4,8.2 .1 .2 \mathrm{~Hz}, 1 \mathrm{H}, \mathrm{Ar}-\mathrm{H}), 5.92$ (d, $J=7.5 \mathrm{~Hz}$, 1H), 4.21 - 4.12 (m, 2H), IR (KBr): $v\left(\mathrm{~cm}^{-1}\right) 2838(\mathrm{C}-\mathrm{H}$ str. in aromatic ring $), 1722(\mathrm{C}=\mathrm{O}$ of thiazolidinone $)$, 1630 (-C=C- str. in aromatic ring), $806(\mathrm{C}-\mathrm{N}-$ str. in s-triazine), MS (70 eV) m/z: $341.7\left[\mathrm{M}^{+}, 100 \%\right], 343.7$ [M+2, $66.66 \%$ ], 345.7 [M+4, $11.11 \%$ ], anal. calcd for $\mathrm{C}_{12} \mathrm{H}_{8} \mathrm{Cl}_{2} \mathrm{~N}_{4} \mathrm{O}_{2} \mathrm{~S}: \mathrm{C}, 42.00 ; \mathrm{H}, 2.35 ; \mathrm{N}, 16.33: \mathrm{S}, 9.34$; found: C, 41.90; H, 2.10; N, 16.10: S, 9.20\%.

3-(4,6-dichloro-1,3,5-triazin-2-yl)-2-(3-methoxy phenyl)thiazolidin-4-one (4j), pale yellow solid, yield$80 \%$, mp- 141-143 ${ }^{\circ},{ }^{1} \mathrm{H}$ NMR (400 MHz, $\mathrm{CDCl}_{3}$ ): $\delta$ (ppm) 8.15 (s, 1H, Ar-H), 7.93 (m, 1H, Ar-H), 7.60 (m, $1 \mathrm{H}, \mathrm{Ar}-\mathrm{H}), 7.46(\mathrm{~m}, 1 \mathrm{H}, \mathrm{Ar}-\mathrm{H}), 5.37(\mathrm{~d}, J=7.1 \mathrm{~Hz}, 1 \mathrm{H})$, $3.75(\mathrm{~s}, 3 \mathrm{H}), 3.38(\mathrm{~d}, 2 \mathrm{H})$; IR (KBr): $v\left(\mathrm{~cm}^{-1}\right) 2834(\mathrm{C}-\mathrm{H}$ str. in aromatic ring), $1717(\mathrm{C}=\mathrm{O}$ of thiazolidinone $)$, $1625(-\mathrm{C}=\mathrm{C}-$ str. in aromatic ring), $806(\mathrm{C}-\mathrm{N}-$ str. in s-triazine), MS (70 eV) m/z: 355.7 [M+1 $100 \%$ ], 357.7 [M+2, $66.66 \%], 359.7$ [M+4, $11.11 \%$ ], anal. calcd for $\mathrm{C}_{13} \mathrm{H}_{10} \mathrm{Cl}_{2} \mathrm{~N}_{4} \mathrm{O}_{2} \mathrm{~S}: \mathrm{C}, 43.71 ; \mathrm{H}, 2.82 ; \mathrm{N}, 15.68: \mathrm{S}, 8.98$; found: $\mathrm{C}, 43.50 ; \mathrm{H}, 2.75 ; \mathrm{N}, 15.42$ : $\mathrm{S}, 8.60 \%$. 3-(4,6-dichloro-1,3,5-triazin-2-yl)-2-(2,5-difluoro phenyl)thiazolidin-4-one (4k), pale yellow solid, yield$75 \%$, mp- 178-180.${ }^{1} \mathrm{H}$ NMR (400 MHz, $\left.\mathrm{CDCl}_{3}\right) \delta$ (ppm): 7.72 (dd, $J=6.3,2.5 \mathrm{~Hz}, 1 \mathrm{H}), 7.39$ (dd, $J=8.6$, $4.5,2.5 \mathrm{~Hz}, 1 \mathrm{H}), 6.85(\mathrm{dd}, J=9.5,8.6 \mathrm{~Hz}, 1 \mathrm{H}), 5.80$ $(\mathrm{s}, 1 \mathrm{H}), 4.15(\mathrm{~m}, 2 \mathrm{H}), \mathrm{IR}(\mathrm{KBr}): v\left(\mathrm{~cm}^{-1}\right) 2825(\mathrm{C}-\mathrm{H}$ str. in aromatic ring), $1725(\mathrm{C}=\mathrm{O}$ of thiazolidinone $)$, $1650(-\mathrm{C}=\mathrm{C}-\mathrm{str}$. in aromatic ring), 813(C-N- str. in s-triazine) $\mathrm{cm}^{-1}$, MS (70 eV) $\mathrm{m} / z: 361.1\left[\mathrm{M}^{+}, 100 \%\right]$, $363.1[\mathrm{M}+2,66.66 \%], 365.1$ [M+4, $11.11 \%]$, anal. calcd for $\mathrm{C}_{12} \mathrm{H}_{6} \mathrm{Cl}_{2} \mathrm{~F}_{2} \mathrm{~N}_{4} \mathrm{OS}$ : C, 39.69; H, 1.67; N, 15.43: S, 8.83; found: C, 39.50; H, 1.44; N, 15.33; S, 8.32\%.

3-(3-(4,6-dichloro-1,3,5-triazin-2-yl)-4-oxo thiazolidin-2-yl)benzonitrile (41), pale yellow solid, yield- $78 \%$, mp- $145-147^{\circ},{ }^{1} \mathrm{H}$ NMR $(400 \mathrm{MHz}$, $\left.\mathrm{CDCl}_{3}\right): \delta(\mathrm{ppm}) 7.94(\mathrm{~s}, 1 \mathrm{H}, \mathrm{Ar}-\mathrm{H}), 7.59(\mathrm{~s}, 1 \mathrm{H}, \mathrm{Ar}-$ $\mathrm{H})$, 7.34-7.21 (m, 1H, Ar-H), 7.04-7.02 ( $\mathrm{m}, 1 \mathrm{H}$, Ar$\mathrm{H}), 5.78(\mathrm{~s}, 1 \mathrm{H}), 3.40-3.36(\mathrm{~m}, 2 \mathrm{H}), \mathrm{IR}(\mathrm{KBr}): v\left(\mathrm{~cm}^{-1}\right)$ 2835 (C-H str. in aromatic ring), $1724(\mathrm{C}=\mathrm{O}$ of thiazolidinone), 1640 (-C=C-str. in aromatic ring), 806 (C-N- str. in s-triazine), MS $(70 \mathrm{eV}) \mathrm{m} / \mathrm{z}: 350.8\left[\mathrm{M}^{+}\right.$, $100 \%], 352.8$ [M+2, $66.66 \%$ ], 354.8 [M+4, $11.11 \%$ ], anal. calcd for $\mathrm{C}_{13} \mathrm{H}_{7} \mathrm{Cl}_{2} \mathrm{~N}_{5} \mathrm{OS}: \mathrm{C}, 44.33 ; \mathrm{H}, 2.00 ; \mathrm{N}$, 19.88; S, 9.10; found: C, 44.10; H, 1.90; N, 19.56: S, $8.89 \%$.

\section{Antiinflammatory assay:}

Proinflammatory cytokine production by lipopolysaccharide (LPS) in THP-1 cells was measured according to the method described by Hwang et al. ${ }^{[29]}$. During the assay, THP-1 cells were cultured in RPMI 1640 culture medium (Gibco BRL, Pasley, UK), containing $100 \mathrm{U} / \mathrm{ml}$ penicillin and $100 \mathrm{mg} / \mathrm{ml}$ streptomycin containing $10 \%$ foetal bovine serum (JRH). Cells were differentiated with phorbol myristate acetate (PMA, Sigma). Following cell plating, the test compounds 3-(4,6-dichloro-1,3,5-triazin-2-yl)2-phenylthiazolidin-4-one (4a-1) in $0.5 \%$ dimethyl sulfoxide were added to each well separately and the plate was incubated for $30 \mathrm{~min}$ at $37^{\circ}$. Finally, LPS (Escherichia coli 0127:B8, Sigma Chemical Co., St. Louis, MO) was added, at a final concentration of 1 $\mu \mathrm{g} / \mathrm{ml}$ in each well. Plates were further incubated at $37^{\circ}$ for $24 \mathrm{~h}$ in $5 \% \mathrm{CO}_{2}$. After incubation, supernatants were harvested and assayed for tumor necrosis factor (TNF)- $\alpha$ and IL- 6 by ELISA as described by the manufacturer (BD Biosciences). The percent inhibitions were measured at $10 \mu \mathrm{M}$ concentration. The dexamethasone was used as standard drugs. The results are tabulated in Table 1. 


\begin{tabular}{|c|c|c|c|}
\hline \multirow{3}{*}{$\begin{array}{l}\text { Compounds } \\
(4 a-1)\end{array}$} & \multirow{3}{*}{ Substituent R } & \multirow{2}{*}{\multicolumn{2}{|c|}{$\%$ Inhibition at $10 \mu \mathrm{M}$}} \\
\hline & & & \\
\hline & & TNF- $a$ & IL-6 \\
\hline $4 a$ & $\mathrm{H}$ & 12 & 20 \\
\hline $4 b$ & 4-OMe & 32 & 29 \\
\hline $4 c$ & $2-\mathrm{F}$ & 70 & 76 \\
\hline $4 d$ & $2-\mathrm{F}, 4-\mathrm{Br}$ & 54 & 57 \\
\hline $4 \mathrm{e}$ & $4-\mathrm{Br}$ & 22 & 23 \\
\hline $4 f$ & $2-\mathrm{F}, 5-\mathrm{Cl}$ & 60 & 67 \\
\hline $4 g$ & 3-Me & 10 & 18 \\
\hline $4 \mathrm{~h}$ & $4-\mathrm{NO}_{2}$ & 15 & 20 \\
\hline $4 i$ & $2-\mathrm{OH}$ & 22 & 29 \\
\hline $4 j$ & 3-OMe & 45 & 54 \\
\hline $4 k$ & $2-F, 5-F$ & 72 & 79 \\
\hline $4 \mathrm{l}$ & $5-\mathrm{CN}$ & 30 & 29 \\
\hline $\begin{array}{l}\text { Reference } \\
\text { drug }\end{array}$ & $\begin{array}{l}\text { Dexamethasone } \\
(1 \mu \mathrm{M})\end{array}$ & 75 & 81 \\
\hline
\end{tabular}

\section{Molecular docking:}

Literature and RCSB Protein Data Bank (www.rcsb. org) survey revealed that no TNF- $\alpha$ trimer has been cocrystallized with small molecules; however a TNF- $\alpha$ dimer structure binding with a small molecule SPD304 is available. Therefore, for molecular docking, the 3D-structure of human TNF- $\alpha$ with antagonist SPD-304 (PDB code: 2AZ5, resolution: $2.1 \AA$ ) was downloaded and subjected to minimization using UCSF Chimera (http://www.cgl.ucsf.edu/chimera/). Molecular docking was performed using NRG Suite ${ }^{[30,31]}$, a python and $\mathrm{C}++$ based PyMOL plugin, which detects surface cavities in proteins and use them as target binding-sites for docking simulations with the aid of FlexAID ${ }^{[30,31]}$. In the present work, following default settings were used to get optimum performance of NRGsuite: binding sites input method, spherical shape; spacing of three dimensional grid: $0.375 \AA$; side chain flexibility- no; ligand flexibility- yes; ligand pose as reference- yes; constraints- no; HET groupsincluded water molecules; vander Walls permeability0.1 ; solvent types- no type; number of chromosomes1000; number of generations- 1000; fitness modelshare; reproduction model- population boom; number of TOP complexes- 10 .

\section{Pharmacophore modelling:}

For pharmacophore modelling, PyMOL 2.1 ${ }^{[32]}$ along with its plugin LIQUID and LigFit were used with default settings. The molecules (three most active) were aligned to get consensus pharmacophore model.

\section{RESULTS AND DISCUSSION}

The synthesis of different 3-(4,6-dichloro-1,3,5triazin-2-yl)-2-phenyl thiazolidin-4-ones (4a-1) were successfully achieved by the reaction between aromatic aldehyde, 4,6-dichloro-1,3,5-triazin-2-amine and ethyl 2-mercaptoacetate in dry ethanol and yield of the product obtained was from 76 to $86 \%$. The antiinflammatory activity of 3-(4,6-dichloro-1,3,5triazin-2-yl)-2 phenylthiazolidin-4-ones were recorded on the basis of reference standard drug dexamethasone and tabulated in Table 1. Some of the compounds showed highest (4c, 4f and $4 \mathrm{k}$ ) antiinflammatory activity. To understand the structural feature, which steer the activity profile, all the molecules were docked in the active site of TNF- $\alpha$. For the sake of convenience and representation, herein, we present the docking pose for most active molecule 4k. Fig. 3 shows the molecular docking poses for molecule $4 \mathrm{k}$, as a representative, within the active site of the enzyme TNF- $\alpha$ and fig. 4 shows the consensus pharmacophore model for most active molecules after alignment.

In this multicomponent reaction (fig. 2), synthesis of 3-(4,6-dichloro-1,3,5-triazin-2-yl)-2-phenyl thiazolidin-4-ones was obtained in good to better yield. This cyclo-condensation multicomponent reaction starts

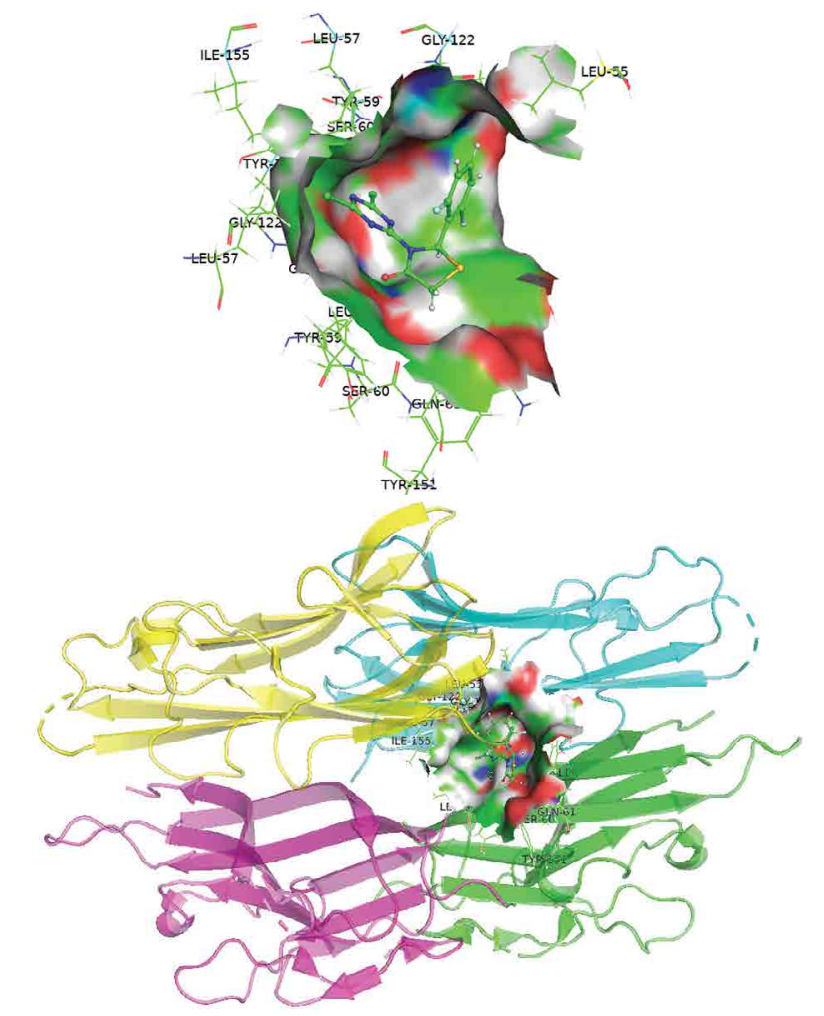

Fig. 3: Docking poses for molecule 4k

Molecular docking poses for the representative molecule $4 k$ within the active site of the enzyme TNF- $\alpha$ 


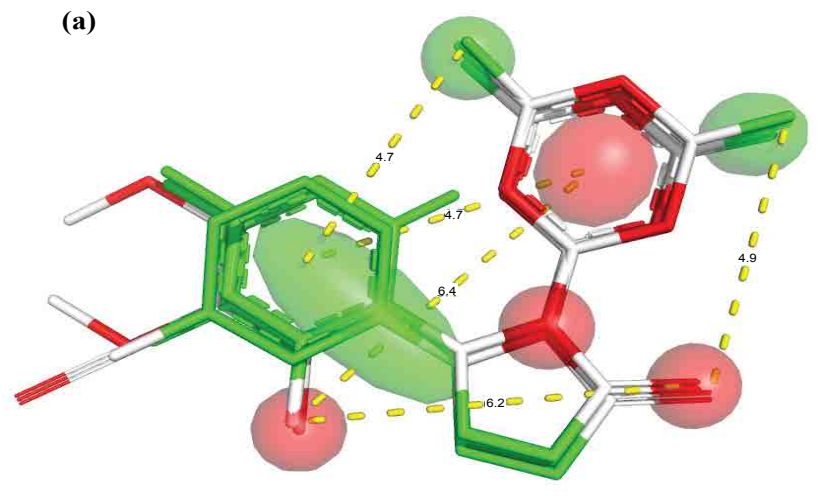

(b)

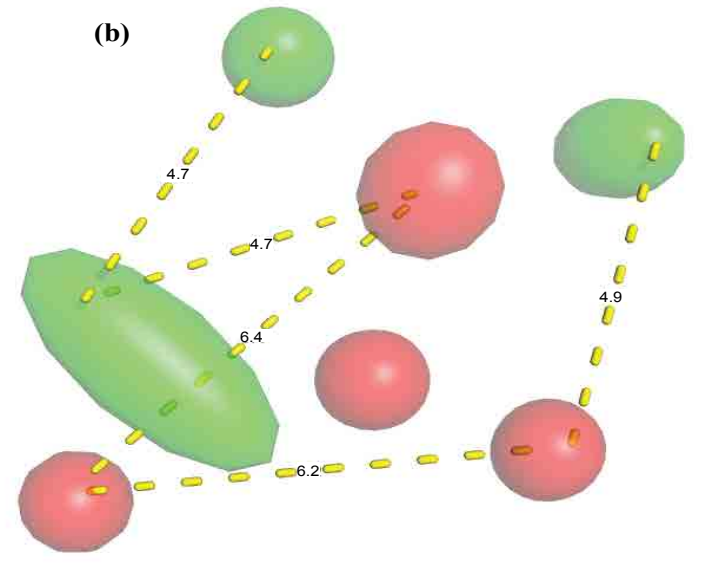

Fig. 4: Consensus pharmacophore model for most active molecules after alignment

(a) Pharmacophore model with molecules as visible, (b) pharmacophore model with molecules made invisible (green contour: hydrophobic, red contour: H-bond acceptor)

with reaction of 4,6-dichloro-1,3,5-triazin-2-amine and aldehyde, which on further reaction with ethyl 2-mercaptoacetate gives the cyclic intermediates and finally results into product. The mechanism of this cyclo-condensation multicomponent reaction is shown is fig. 5 .

From antiinflammatory activity data of 3-(4,6dichloro-1,3,5-triazin-2-yl)-2-phenylthiazolidin-4ones (Table 1), it was observed that the compounds $4 \mathrm{c}$, $4 \mathrm{f}$, and $4 \mathrm{k}$ were found to be comparatively active as TNF- $\alpha$ and IL- 6 inhibitor (up to $60-72 \%$ TNF- $\alpha$ and 67-79\% IL-6 inhibitory activity), while compounds $4 \mathrm{k}$ (72\% and $79 \%$ ) exhibiting highest inhibition against TNF- $\alpha$ and IL-6, respectively at $10 \mu \mathrm{M}$. It is to be noted that the compound found to be equieffective to $1 \mu \mathrm{M}$ dexamethasone. The compounds $4 \mathrm{~d}$ and $4 \mathrm{j}$ exhibited moderate activity (45-57 \% inhibition) while other compounds exhibited low $(4 \mathrm{~b}, 4 \mathrm{e}, 4 \mathrm{i}, 4 \mathrm{l})$ to very low $(4 \mathrm{a}, 4 \mathrm{~g}, 4 \mathrm{~h})$ activity at same level of concentration. The substitution of functional group such as $-\mathrm{OCH}_{3}$, $-\mathrm{OH},-\mathrm{Cl}$ and $-\mathrm{F}$ showed significant activity. The antiinflammatory activity data shows that presence of fluorine group at $\mathrm{C}-2$ and $\mathrm{C}-5$ of phenyl ring plays an important role in the activity. The presence of ' $F$ ' group at C-5 enhances the antiinflammatory activity (4k). Because of the presence of chlorine instead of fluorine at C-5 compound (4f) little bit reduces the activity. Further, it was observed that the presence of fluorine at position-2, bromine at position-4 and methoxy group at position-3 on phenyl ring showed moderate activity. Interestingly, the presence of other functional groups on different position on phenyl such as $4-\mathrm{OMe}, 4-\mathrm{Br}$, 3-Me, 2-OH, 4- $\mathrm{NO}_{2}$ and 5-CN exhibits low to very low activity.

It was revealed from these SAR studies that the presence of a halide (electron donating deactivating group) $-\mathrm{F}$ and $-\mathrm{Cl}$ at $\mathrm{C}-5$ position tolerates the procytokine activity. It was found that fluorine imparts the special characteristics that enhance therapeutic efficiency and improved pharmacological properties in active molecules. Thus, the compound $4 \mathrm{k}$ and $4 \mathrm{c}$ were found to be potential antiinflammatory agents amongst the series of compounds studied.

Themolecular docking analysis for most active molecule $4 \mathrm{k}$ revealed that the molecules have hydrophobic and polar interactions with the receptor residues (fig. 3). From the docking pose, it appeared that the molecule $4 \mathrm{k}$ adopted a claw-like shape and presented at the interface of two chains of the enzyme. Its aromatic ring was found to be responsible for hydrophobic interactions with nearby residues viz. Leu55, Leu120, and Tyr59. The importance of hydrophobic part of molecules has also been highlighted by the consensus pharmacophoric analysis (fig. 4).

The two chlorine atoms on the triazine ring also favors hydrophobic interaction with the residues viz. SER60, Tyr151, Gly122, and Leu120. Therefore, presence of two chlorine atoms is a good feature for retentions in the future optimizations. The five membered thiazolidinone rings has contributed as a bridge and added additional flexibility to adopt the appropriate conformation inside the active site of the receptor. This ring is closer to chain $\mathrm{B}$ of the receptor and responsible for polar interactions with the following residues of chain B viz. Ser60 and Gln61. It appeared that docking and pharmacophore analyses provide similar results and highlight hydrophobic as important feature for activity optimization.

In the present work, all molecules (4a-4l) were subjected to molecular docking in the active site of human TNF- $\alpha$. Table 2 contains the docking scores for 


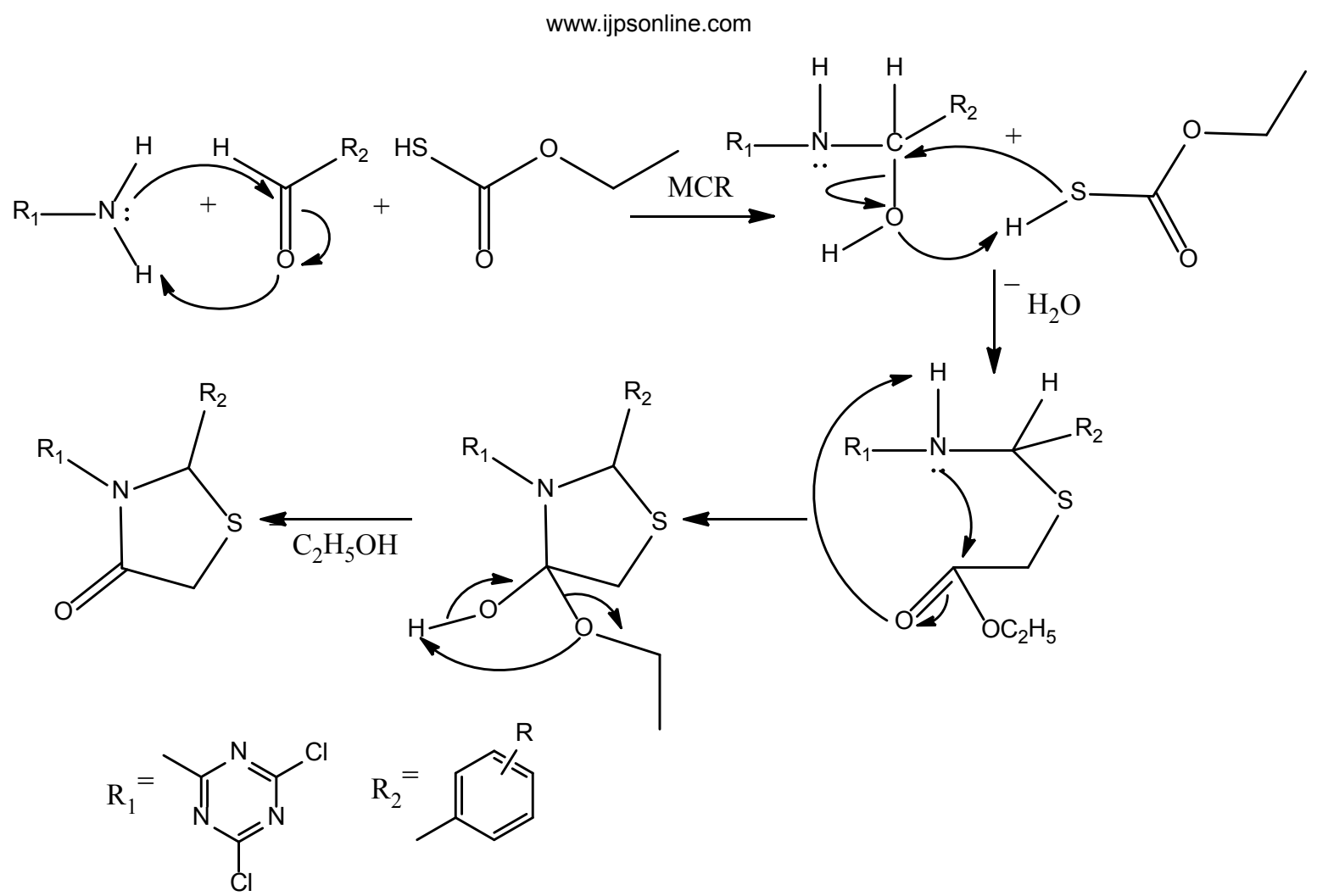

Fig. 5: Mechanism of the multicomponent reaction

Multicomponent reaction involved in the synthesis of aryl substituted 3-(4,6-dichloro-1,3,5-triazin-2-yl) thiazolidin-4-ones

TABLE 2: DOCKING SCORES FOR MOLECULES 4A-4L AND ORIGINAL LIGAND (SPD304)

\begin{tabular}{|c|c|c|}
\hline ID & SMILES & Docking score \\
\hline $4 a$ & Clc1nc(Cl)nc(N2C $(=0) \operatorname{CSC} 2 \operatorname{cocccc} 2) n 1$ & -5.9930043 \\
\hline $4 \mathrm{~b}$ & $\mathrm{Clc} 1 \mathrm{nc}(\mathrm{Cl}) \mathrm{nc}(\mathrm{N} 2 \mathrm{C}(=0) \operatorname{CsC} 2 \mathrm{c} 2 \mathrm{ccc}(\mathrm{OC}) \mathrm{cc} 2) \mathrm{n} 1$ & -6.1437421 \\
\hline 4c & $\mathrm{Clc} 1 \mathrm{nc}(\mathrm{Cl}) \mathrm{nc}(\mathrm{N} 2 \mathrm{C}(=0) \operatorname{CsC} 2 \mathrm{c} 2 \mathrm{c}(\mathrm{F}) \mathrm{cccc} 2) \mathrm{n} 1$ & -5.9163222 \\
\hline $4 \mathrm{~d}$ & $\operatorname{Brc} 1 \mathrm{cc}(\mathrm{F}) \mathrm{c}(\mathrm{C} 2 \mathrm{~N}(\mathrm{c} 3 \mathrm{nc}(\mathrm{Cl}) \mathrm{nc}(\mathrm{Cl}) \mathrm{n} 3) \mathrm{C}(=0) \mathrm{CS} 2) \mathrm{cc} 1$ & -6.1437573 \\
\hline $4 e$ & $\operatorname{Brc} 1 \operatorname{ccc}(\mathrm{C} 2 \mathrm{~N}(\mathrm{c} 3 \mathrm{nc}(\mathrm{Cl}) \mathrm{nc}(\mathrm{Cl}) \mathrm{n} 3) \mathrm{C}(=0) \mathrm{CS} 2) \mathrm{cc} 1$ & -5.9886723 \\
\hline $4 f$ & $\mathrm{Clc} 1 \mathrm{nc}(\mathrm{Cl}) \mathrm{nc}(\mathrm{N} 2 \mathrm{C}(=0) \mathrm{CSC} 2 \mathrm{c} 2 \mathrm{c}(\mathrm{Cl}) \operatorname{cccc} 2 \mathrm{~F}) \mathrm{n} 1$ & -6.1999474 \\
\hline $4 \mathrm{~g}$ & $\mathrm{Clc} 1 \mathrm{nc}(\mathrm{Cl}) \mathrm{nc}(\mathrm{N} 2 \mathrm{C}(=0) \mathrm{CsC} 2 \mathrm{c} 2 \mathrm{cc}(\mathrm{C}) \mathrm{ccc} 2) \mathrm{n} 1$ & -6.0782661 \\
\hline $4 \mathrm{~h}$ & $\operatorname{Clc} 1 n c(C l) n c(N 2 C(=0) \operatorname{CsC} 2 \operatorname{coccc}([N+](=0)[0-]) c c 2) n 1$ & -6.4120026 \\
\hline $4 i$ & $\mathrm{Clc} 1 \mathrm{nc}(\mathrm{Cl}) \mathrm{nc}(\mathrm{N} 2 \mathrm{C}(=0) \operatorname{CsC} 2 \mathrm{c} 2 \mathrm{c}(\mathrm{O}) \mathrm{cccc} 2) \mathrm{n} 1$ & -5.9833794 \\
\hline $4 j$ & $\mathrm{Clc} 1 \mathrm{nc}(\mathrm{Cl}) \mathrm{nc}(\mathrm{N} 2 \mathrm{C}(=0) \operatorname{CsC} 2 \mathrm{c} 2 \mathrm{cc}(\mathrm{OC}) \mathrm{ccc} 2) \mathrm{n} 1$ & -6.1720862 \\
\hline $4 \mathrm{k}$ & $\mathrm{Clc} 1 \mathrm{nc}(\mathrm{Cl}) \mathrm{nc}(\mathrm{N} 2 \mathrm{C}(=0) \operatorname{CsC} 2 \mathrm{c} 2 \mathrm{c}(\mathrm{F}) \mathrm{cccc} 2 \mathrm{~F}) \mathrm{n} 1$ & -5.9358053 \\
\hline $4 l$ & $\mathrm{Clc} 1 \mathrm{nc}(\mathrm{Cl}) \mathrm{nc}(\mathrm{N} 2 \mathrm{C}(=0) \mathrm{CSC} 2 \mathrm{c} 2 \mathrm{cc}(\mathrm{C \# N}) \mathrm{ccc} 2) \mathrm{n} 1$ & -6.3134403 \\
\hline SPD304 & $\mathrm{FC}(\mathrm{F})(\mathrm{F}) \mathrm{c} 1 \mathrm{cc}(-\mathrm{n} 2 \mathrm{c} 3 \mathrm{c}(\mathrm{c}(\mathrm{C}[\mathrm{N}+](\mathrm{CC}[\mathrm{N}+](\mathrm{CC} 4=\mathrm{C}(\mathrm{O}) \mathrm{c} 5 \mathrm{c}(\mathrm{OC} 4) \mathrm{cc}(\mathrm{C}) \mathrm{c}(\mathrm{C}) \mathrm{c5}) \mathrm{C}) \mathrm{C}) \mathrm{c2}) \mathrm{cccc} 3) \mathrm{ccc} 1$ & -7.6338243 \\
\hline
\end{tabular}

the molecules. For the sake of validation of molecular docking, the original ligand (SPD304) was also docked in the active site of the receptor and its docking score is also tabulated in Table 2. From the Table 2, it is clear that the original ligand (SPD304) has better binding with the receptor than the molecules $4 \mathrm{a}-41$. Therefore, future modifications are required to increase selectivity and activity profile of triazine thiazolidinone derivatives, synthesized in the present work, for human TNF- $\alpha$.

In summary, a series of 3-(4,6-dichloro-1,3,5-triazin-2yl)-2-phenylthiazolidin-4-one derivatives (4a-1) were synthesized in good to better yield by condensation cyclisation reaction between substituted aromatic aldehyde, 4,6-dichloro-1,3,5-triazin-2-amine and ethyl 2-mercaptoacetate using dry ethanol. The SAR study, suggested that the halogenated electron-withdrawing groups imparted greater antiinflammatory activity from the title hybrid skeleton. Furthermore, molecular docking and pharmacophore analyses provided similar results and highlighted hydrophobic moieties as prominent features for activity optimization.

\section{Conflict of interest:}

Authors have no conflict of interest. 


\section{REFERENCES}

1. Centers for Disease Control and Prevention. Antibiotic resistance threats in the United States, 2013. Atlanta, GA: CDC, 2016 [cited 25 March 2019]. Available from: http:// www.cdc.gov/drugresistance/threat-report-2013/.

2. High levels of antibiotic resistance found worldwide, new data shows, news release on $29^{\text {th }}$ January, 2018 [cited 12 March 2019] Available from: https://www.who.int/mediacentre/ news/releases/2018/antibiotic-resistance-found/en/.

3. Levy SB, Marshall B. Antibacterial resistance worldwide: causes, challenges and responses. Nat Med 2004;10(12 suppl):S122-9.

4. Demain AL, Sanchez S. Microbial drug discovery: 80 years of progress. J Antibiot 2009;62:5-16.

5. Gootz TD. The global problem of antibiotic resistance. Crit Rev Immunol 2010;30(1):79-93.

6. Niccolai D, Tarsi L, Thomas RJ. The renewed challenge of antibacterial chemotherapy. Chem Commun 1997;24:233342.

7. Sadek B, Al-Tabakha MM, Fahelelbom KM. Antimicrobial Prospect of Newly Synthesized 1,3-Thiazole Derivatives. Molecules 2011;16(11):9386-96.

8. Haroun M, Tratrat C, Tsolaki E, Geronikaki A. ThiazoleBased Thiazolidinones as Potent Antimicrobial Agents. Design, Synthesis and Biological Evaluation. Comb Chem High Throughput Screen 2016;19(1):51-7.

9. Palekar VS, Damle AJ, Shukla SR. Synthesis and antibacterial activity of some novel bis-1,2,4-triazolo[3,4-b]-1,3,4thiadiazoles and bis-4-thiazolidinone derivatives from terephthalic dihydrazide. Eur J Med Chem 2009;44:5112-6.

10. Hamama WS, Ismail MA, Shaaban S, Zoorob HH. Progress in the chemistry of 4- thiazolidinones. J Heterocycl Chem 2008;45:939-56.

11. Kumar M, Ramasamy K, Mani V, Mishra RK, Majeed ABA, Clercq ED, Narasimhan B. Synthesis, antimicrobial, anticancer, antiviral evaluation and QSAR studies of 4-(1-aryl2-oxo-1,2-dihydro-indol-3-ylideneamino)-N-substituted benzene sulfonamides. Arab J Chem 2014;7:396-408.

12. Kaminskyy D, Bednarczyk-Cwynar B, Vasylenko O, Kazakova O, Zimenkovsky B, Zaprutko L, Lesyk R. Synthesis of new potential anticancer agents based on 4-thiazolidinone and oleanane scaffolds. Med Chem Res 2011;21:3568-80.

13. Samadhiya P, Sharma R, Srivastava SK, Srivastava SD. Synthesis and biological evaluation of 4-thiazolidinone derivatives as antitubercular and antimicrobial agents. Arab J Chem 2014;7:657-65.

14. Kaur MS, Kaur R, Bhatia R, Kumar K, Singh V, Shankar R, et al. Synthetic and medicinal perspective of thiazolidinones: a review. Bioorg Chem 2017;75:406-23.

15. Mistry KM, Desai KR. Synthesis of novel heterocyclic 4-thiazolidinone derivatives and their antibacterial activity. E-J Chem 2004;1(4):189-93.

16. Taranalli AD, Bhat AR, Srinivas S, Saravanan E.
Antiinflammatory, analgesic and antipyretic activity of certain thiazolidinones. Indian J Pharm Sci 2008;70(2):159-64.

17. Wang S, Zhao Y, Zhu W, Liu Y, Guo K, Gong P. Synthesis and anticancer activity of indolin-2-one derivatives bearing the 4-thiazolidinone moiety. Arch Pharm 2012;345:73-80.

18. Balzarini J, Orzeszko-Krzesińska B, Maurin JK, Orzeszko A. Synthesis and anti-HIV studies of 2- and 3-adamantylsubstituted thiazolidin-4-ones. Eur J Med Chem 2009;44:30311.

19. Srinivas A, Nagaraj A, Reddy CS. Synthesis and biological evaluation of novel methylene-bisthiazolidinone derivatives as potential nematicidal agents. J Heterocycl Chem 2008;45:9991003.

20. Patel RV, Keum YS, Park SW. Medicinal chemistry discoveries among 1,3,5-triazines: recent advances (20002013) as antimicrobial, anti-TB, anti-HIV and antimalarials. Mini Rev Med Chem 2014;14(9):768-89.

21. Gahtori P, Singh BK, Das A. Synthesis of phenyl-1,3-thiazole substituted amino s-triazines as antibacterial agents. Dhaka Univ J Pharm Sci 2009;7:107-11.

22. Kumar S, Bhat HR, Kumawat MK, Singh UP. Design and one-pot synthesis of hybrid thiazolidin-4-one-1,3,5-triazines as potent antibacterial agents against human disease-causing pathogens. New J Chem 2013;37:581-4.

23. Thabet HK, Ubeid MT, El-Feky SA. Synthesis of novel 4-thiazolidinones linked by an aryl spacer to a 1,2,4-triazine moiety. J Chem Res 2015; 39:567-73.

24. Patel D, Patel R, Kumari P, Patel NB. Synthesis of s-triazinebased thiazolidinones as antimicrobial agents. Z Naturforsch 2012;67(3-4):108-22.

25. Rana AM, Trivedi P, Desai KR, Jauhari S. Novel S-triazine accommodated 5-benzylidino-4-thiazolidinones: synthesis and in vitro biological evaluations. Med Chem Res 2014;23:4320-36.

26. Hamama WS, Ismail MA, Shaaban S, H.H. Zoorob. Synthesis and biological evaluation of some new Thiazolo[3,2-a][1,3,5] triazine derivatives. Med Chem Res 2011;21:2615-23.

27. Patel D, Kumari P, Patel N. Synthesis and biological evaluation of some thiazolidinones as antimicrobial agents. Eur J Med Chem 2012;48:354-62.

28. Shinde RS. Facile multicomponent synthesis of thiazolidinone based triazines derivatives as new potential anti-microbial agent. J Ultra Sci Phys Sci 2018; 30:49-56.

29. Hwang C, Gatanaga M, Granger GA, Gatanaga T. Mechanism of release of soluble forms of tumor necrosis factor/ lymphotoxin receptors by phorbol myristate acetate-stimulated human THP-1 cells in vitro. J Immunol 1993;151:5631-8.

30. Gaudreault F, Najmanovich RJ. FlexAID: Revisiting docking on non-native-complex structures. J Chem Inf Model 2015; 55:1323-36.

31. Gaudreault F, Morency LP, Najmanovich RJ. NRGsuite: a PyMOL plugin to perform docking simulations in real time using FlexAID. Bioinformatics 2015;31:3856-8.

32. Yuan S, Chan HCS, Hu Z. Using PyMOL as a platform for computational drug design. Comput Mol Sci 2017;7:e1298. 